\title{
Acquired Long QT Syndrome in Severe COVID-19 Patient Treated with Chloroquine Azithromycin and Lopinavir/ Ritonavir: Simple Monitoring, Reducing Doses or Stopping Treatment?
}

\section{Najout $\mathrm{H}^{1 *}$, Elkoundi $\mathrm{A}^{1}$, Kartite $\mathbf{N}^{1}$, Fihri $\mathbf{Y}^{2}$ and Balkhi $\mathbf{H}^{1}$ \\ ${ }^{1}$ Anesthesia-Critical Care Department, Mohamed V Military Training Hospital, Rabat, Morocco \\ ${ }^{2}$ Department of Cardiology, Mohamed V Military Training Hospital, Rabat, Morocco \\ *Corresponding author: Hamza Najout, Anesthesia- Critical Care Department, Mohamed V Military Training Hospital, Rabat, Morocco}

Received: September 27, 2021; Accepted: October 23, 2021; Published: October 30, 2021

\section{Introduction}

Some drugs used to treat COVID-19 can cause QT prolongation.

QT prolongation is associated with high risk of torsade de pointes, ventricular arrhythmia and sudden death.

Early identification is crucial to ovoid devastating outcomes.

\section{Case Presentation}

A 45-year-old man with COVID-19 confirmed by RT-PCR was admitted to ICU for the treatment of hypoxemic respiratory failure requiring mechanical ventilation.

He was treated with Chloroquine Hydrochloride 200mg twice a day, Azithromycin 250mg per day, Lopinavir/Ritonavir 400/100mg twice a day and low molecular-weight heparin $0.6 \mathrm{ml}$ twice a day. The Biological assessment showed hypokalemia at $2.9 \mathrm{mmol} / \mathrm{L}$ and the ECG showed long QT syndrome. Potassium plasma level was corrected through jugular venous catheter and a second

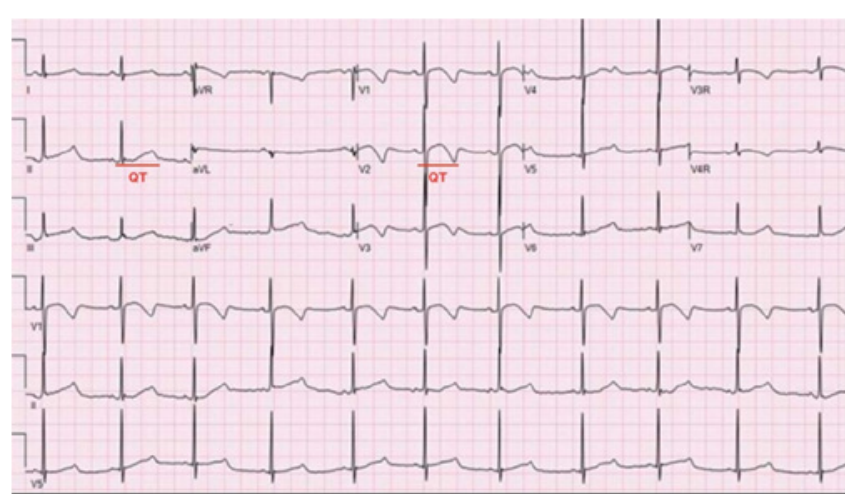

Figure 1: (Before) Electrocardiogram shows long QT (QT=480 ms).

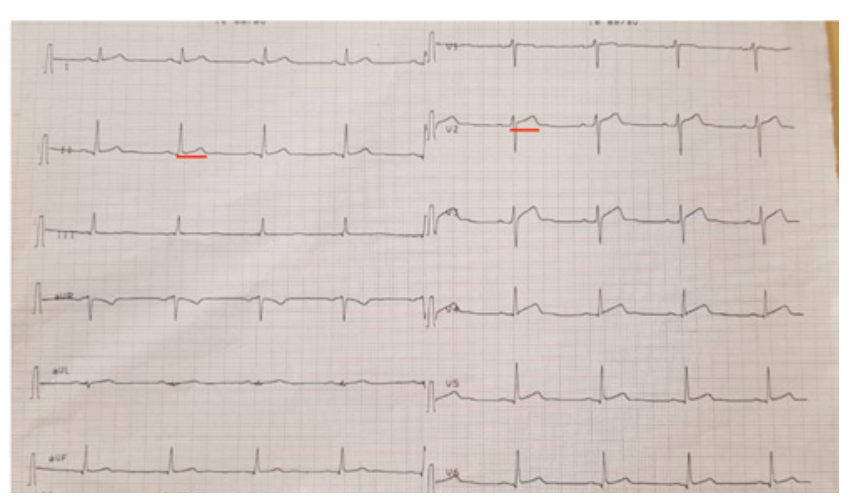

Figure 2: (After) Electrocardiogram shows normal QT space (QT=440 ms).

ECG showed the persistence of long QT space at 480ms (Figure 1). Electrocardiography and troponin plasma level were normal. Drugs doses were reduced to the half with strict control of calcium, magnesium and potassium plasma levels. An ECG was performed twice a day and QT space was normalized within 6 days (Figure 2). Thereafter, we have resumed normal doses and an ECG was daily realized until the patient was discharged on the twenty fifth day.

SARS-Cov-2, is known for its respiratory tropism causing also a state of hyper coagulopathy, especially in critically ill patients [1]. Our case shows the existence of a vacuum in the literature about the management of acquired long QT syndrome in severe and polypharmacy patients with COVID-19.

\section{Authors Contributions}

Hamza Najout: Prepared and wrote this manuscript. Abdelghafour Elkoundi: Reviewed this manuscript. Noureddine Kartite: Reviewed this manuscript. Youssef El Fihri: Responsible for clinical consultation. Hicham Balkhi: Revision of this manuscript.
Austin J Clin Case Rep - Volume 8 Issue 9 - 2021 ISSN : 2381-912X | www.austinpublishing group.com Najout et al. (C) All rights are reserved
Citation: Najout H, Elkoundi A, Kartite N, Fihri Y and Balkhi H. Acquired Long QT Syndrome in Severe COVID-19 Patient Treated with Chloroquine Azithromycin and Lopinavir/Ritonavir: Simple Monitoring, Reducing Doses or Stopping Treatment?. Austin J Clin Case Rep. 2021; 8(9): 1232. 


\section{References}

COVID-19: What we know so far. J Chin Med Assoc. 2020; 83: 534-536.

1. Lu CC, Chen MY, Lee WS, Chang YL. Potential therapeutic agents against 